\title{
Cuestionario de Fallos de Memoria de la Vida Cotidiana (MFE). Análisis de factores con población española
}

\author{
Pedro Montejo ${ }^{1 *}$, Mercedes Montenegro ${ }^{12}$, Manuel J. Sueiro-Abad ${ }^{2}$ y Evelio Huertas ${ }^{2}$ \\ 1 Centro de Prevención del Deterioro Cognitivo. Instituto de Salud Pública. Madrid Salud. Ayuntamiento de Madrid. Madrid (España) \\ 2 Facultad de Psicología. Universidad Complutense de Madrid. Madrid (España)
}

Resumen: Uno de los instrumentos más utilizados para valorar los olvidos
cotidianos es el Cuestionario de Fallos de Memoria de la Vida Cotidiana
(MFE). Los objetivos del presente estudio fueron: a) examinar la estructura
factorial del MFE, b) explorar las relaciones de los factores resultantes con
el rendimiento objetivo de memoria (Listas de Palabras y Escenas de la Es-
cala de Memoria de Wechsler - III), el estado de ánimo y la ansiedad (Esca-
la de Depresión y Ansiedad de Goldberg) y con dos preguntas generales
sobre quejas de memoria. Los datos se recogieron de una muestra de 647
adultos jóvenes (19-64 años). Los resultados mostraron la existencia de una
estructura de tres factores, que explicaban el 29.3 \% de la varianza: Recuer-
do de Actividades, Monitorización de la Comunicación y Reconocimiento.
Los dos primeros factores mostraron una correlación positiva más alta en-
tre ellos y una asociación mayor con el estado de ánimo y las quejas genera-
les de memoria. Por el contrario, el factor Reconocimiento presentó una
correlación positiva más alta con la edad y negativa con el rendimiento ob-
jetivo de memoria.
Palabras clave: MFE; olvidos cotidianos; análisis factorial; evaluación de Palabras clave: MFE; olvidos
memoria; memoria cotidiana.
Title: Memory failures of Everyday (MFE): Factor Analysis with Spanish population.

Abstract: One of the most frequently used instruments to study everyday memory failures is the Memory Failures of Everyday Questionnaire (MFE). The objectives of the present study were: a) to examine the factor structure of the MFE, and b) to explore the relationship of the resulting factors with performance in tests of objective memory (Word Lists and Family Pictures of the Wechsler Memory Scale-III) and mood state (Goldberg Depression and Anxiety Scale), as well as with two general questions about memory complaints. Data were collected from a sample of 647 young adults (19-64 years). Factor analysis revealed three factors which explained $29.3 \%$ of the total variance: Activities Recall, Communication Monitoring, and Recognition. The first two factors showed a higher positive correlation between them and a higher association with mood state and general memory complaints. In contrast, the recognition factor shows positive correlation with age and negative with objective memory performance.

Key words: MFE; everyday memory failures; factorial analysis; memory assessment; everyday memory.

\section{Introducción}

Los fallos de memoria de la vida cotidiana son un síntoma que preocupa sobre todo a los mayores, pero están cobrando cada vez más importancia entre los adultos jóvenes. Son fallos de memoria, u olvidos cotidianos, olvidar los nombres de las personas, olvidar dónde se ponen las cosas (las gafas, los documentos, las llaves,...), olvidar hacer un recado, olvidar las caras de las personas, lo que hemos hecho últimamente, lo que hemos leído, no recordar un recorrido, etc. Todos ellos forman parte de la memoria cotidiana cuyo estudio se está desarrollando sobre todo desde hace unas tres décadas (West y Sinnott, 1992). Estos fallos, cuando son expresados por el sujeto, se llaman también quejas de memoria, dado que son una manifestación de la percepción que tiene sobre el rendimiento de su memoria. En este sentido, forman parte de la metamemoria (Hermann, 1982). Las quejas de memoria están incluidas como criterio en la Alteración de la Memoria Asociada a la Edad y en el Deterioro Cognitivo Leve. En este último caso, a ser posible, corroboradas por un informador (Crook et al., 1986; Petersen, 2004). Las quejas de memoria se han relacionado con varios factores, uno de los cuales ha sido el rendimiento real de memoria. Una revisión de Reid y MacLullich (2006) sobre quejas de memoria y rendimiento cognitivo y de memoria indica que la evidencia actual no permite sacar conclusiones claras en este tema. La depresión ha sido el factor que más se ha relacionado con las quejas, tanto en adultos jóvenes como en mayores de 65 años. En un estudio longitudinal, Jorm,

* Dirección para correspondencia [Correspondence address]: Pedro Montejo. C/ Montesa 22, Edificio B, 28006 Madrid (España). E-mail: montejop@madrid.es
Christensen, Korten, Jacob y Henderson (2001) concluyeron que las quejas están asociadas sobre todo a ansiedad y depresión. En el estudio de Ponds, Commissaris y Jolles (1997), los más jóvenes atribuían sus fallos a tensión y problemas emocionales, falta de interés y falta de atención/concentración.

Como consecuencia de la importancia de las quejas, se han desarrollado pruebas para evaluar la memoria cotidiana, tanto desde el punto de vista objetivo como subjetivo. Entre las pruebas de memoria objetiva se encuentran la Batería computarizada de Larrabee y Crook (1988) y el Test Conductual de Memoria Rivermead (RMBT) (Wilson, Cockburn y Baddeley, 1985), que evalúan el rendimiento en el recuerdo de nombres, caras, recorrido, etc.

La valoración subjetiva suele realizarse de dos modos: mediante preguntas generales de quejas de memoria o mediante cuestionarios. Aunque no son equivalentes, unas y otros correlacionan positivamente (Abdulrab y Heun, 2008). Entre las preguntas de quejas, la más utilizada es "¿Tiene usted problemas de memoria?", que se contesta de modo dicotómico (Reid y MacLullich, 2006). Entre los cuestionarios, podrían distinguirse los de olvidos y los específicos de metamemoria. Los de olvidos estudian la frecuencia y/o gravedad de diversos fallos de memoria que se tienen en la vida diaria; suelen ser listados de olvidos. Los cuestionarios de olvidos, cuando son autoadministrados, también evalúan metamemoria, pues reflejan la valoración subjetiva que el sujeto hace de su propia memoria. Sin embargo, también pueden ser respondidos por observadores (familiares, personal clínico u otros), y, en este caso, no valoran metamemoria. Entre los cuestionarios de olvidos, cabe destacar el Cognitive Failures Questionnaire (Broadbent, Cooper, Fitzgerald y 
Parkes, 1982), que ha sido adaptado por García-Martínez y Sánchez-Cánovas (1994); el Memory Complaint Questionnaire (MAC-Q) (Crook, Feher y Larrabee, 1992); el Cuestionario de Fallos de Memoria de la Vida Cotidiana (Memory Failures of Everyday-MFE) de Sunderland, Harris y Gleave (Sunderland, Harris y Gleave, 1984), con 28 ítems sobre situaciones y actividades de la vida diaria; el Autoinforme de Memoria para Ancianos (AMA) (Fernández Ballesteros, Izal y Montorio, 1991), con 21 ítems, y el Cuestionario de Olvidos Cotidianos (COC) (Benedet y Seisdedos, 1996), con 68 preguntas agrupadas en 10 apartados. Los de metamemoria plantean cuestiones sobre las estrategias utilizadas para solucionarlos, la influencia en la autopercepción del individuo, el esfuerzo realizado, la predicción que hacemos respecto a dichos olvidos, etc. Entre estos cabe destacar el Metamemory Questionnaire (Zelinski, Gilewski y Thompson, 1980) y el Metamemory in Adulthood Questionnaire (MLA) de Dixon y Hultsch (1983).

Un tema importante relacionado con los olvidos cotidianos y los cuestionarios que los recogen es la investigación de la estructura factorial que subyace a esos olvidos y el estudio de los procesos de memoria que están implicados en esos factores. Se ha estudiado la estructura factorial de algunos de estos cuestionarios, con resultados diversos. Esta diversidad de resultados tiene que ver con la diferente metodología aplicada: métodos de análisis, muestras de características diversas, preguntas y olvidos diferentes.

El Cuestionario de Fallos de Memoria de la Vida Cotidiana (Memory Failures of Everyday, MFE) fue desarrollado en 1983 (Sunderland, Harris y Baddeley, 1983) y tenía 35 ítems. Posteriormente (Sunderland et al., 1984) se elaboró el de 28 ítems, que es el más utilizado en la actualidad. Ha sido adaptado para población española por García-Martínez y Sánchez-Cánovas (1993). En su origen, se construyó atendiendo a los olvidos que tenían pacientes con daño cerebral traumático, preguntándoles a ellos y a sus familiares. Los mismos autores se basaron en estas entrevistas a pacientes y familiares para modificarlo. Para esta versión modificada, tuvieron en cuenta, entre otros criterios, el poder discriminativo de los ítems. Tomaron 22 ítems del original y añadieron otros 6 nuevos, formando el cuestionario de 28 ítems, que ha sido el más estudiado y utilizado. Las categorías de olvidos que recoge son "hablar, leer y escribir", "nombres y caras", "acciones" y "aprender cosas nuevas". Se contesta mediante una escala Likert con nueve opciones de respuesta para cada cuestión, éstas van desde "Ninguna vez en los últimos 3 meses" a "Más de 1 vez al día" aunque, dada la dificultad de las nueve opciones, otros autores han utilizado menos opciones de respuesta.

El MFE puede valorar la situación actual de los pacientes y su evolución con el tiempo o a causa de un tratamiento. Se ha utilizado en rehabilitación (Quemada et al., 2003), demencia (Seltzer, Vasterling, Hale y Khurana, 1995), esclerosis múltiple (Richardson, 1996), epilepsia (Goldstein y Polkey, 1992), para el estudio de la memoria en niños sin alteraciones y con trastornos del aprendizaje (Drysdale, Shores y Levick, 2004), para el estudio de la memoria subjetiva en mayores (Sunderland, Watts, Baddeley y Harris, 1986), para valorar los resultados del entrenamiento de memoria para mayores (Cavallini, Pagnin y Vecchi, 2003; Montejo, Montenegro, Reinoso, De Andrés y Claver, 1999), etc.

En el estudio de análisis de factores, Sunderland et al. (1984) encontraron un solo factor que explicaba, según el tipo de pacientes o familiares, desde el $39 \%$ de la varianza hasta el $60 \%$ y lo interpretaron indicando que había un factor general subyacente a la memoria cotidiana. GarcíaMartínez y Sánchez-Cánovas (1994) realizaron una adaptación del MFE y encontraron también, mediante análisis de componentes principales, un factor no rotado que explicaba el 27\% de la varianza. Richardson (1996), por el contrario, estudiando pacientes con esclerosis múltiple, encontró 5 factores que explicaban el $62 \%$ de la varianza: fallos en la comunicación receptiva, búsqueda de rutas, despistes, fallos en reconocimiento de caras, fallos en la comunicación expresiva. Cornish (2000), por su parte, encontró 5 factores, tres de ellos similares a los de Richardson (1996). Estos factores explicaban el $49 \%$ de la varianza: recuerdo (olvidar sucesos y memoria prospectiva), monitorización de tareas, control de conversaciones, memoria espacial y memoria para actividades. Efklides et al. (2002) encontraron una estructura de 7 factores que explicaba el $62 \%$ de la varianza: problemas en la memoria prospectiva y en la memoria general, dificultades en el aprendizaje y en la repetición de respuestas, olvidar cambios en la rutina diaria, memoria visuoespacial, memoria semántica, memoria episódica y de caras, y memoria de reconstrucción visual. Royle y Lincoln (2008), por último, han encontrado 3 factores que explican el $62 \%$ de la varianza: memoria general, funciones atencionales, y otro factor con ítems que son dispares y difíciles de interpretar como algo común.

La unidimensionalidad del cuestionario, que encuentran algunos investigadores, viene dada por la existencia de una creencia general de las personas sobre cómo funciona su memoria. La multidimensionalidad indica no sólo los diferentes tipos de memoria implicados en los olvidos en diferentes situaciones sino también otros procesos o factores como la atención o la motivación. Algunos autores tratan de explicar los distintos factores del cuestionario atendiendo a los sistemas teóricos de memoria con un resultado heterogéneo, probablemente debido a la diversidad de las muestras, pues se utilizan sujetos sin patología, otros con Enfermedad de Alzheimer, enfermos con esclerosis múltiple y sus familiares, adultos jóvenes y mayores de 65 años, etc. Muchas de esas condiciones afectan a más de un sistema de memoria, por lo que las quejas relativas a esos sistemas deberían covariar. Además, el cuestionario fue creado recogiendo en los ítems los fallos de memoria u olvidos más frecuentemente observados con fines prácticos, al margen de una conceptualización en términos de sistemas y, por lo tanto, sin un muestreo de ítems relativos a todos y cada uno de los sistemas teóricos. No es de extrañar, pues, que los factores obtenidos no se ajusten a esta estructura teórica. 
Hasta ahora, los trabajos que han estudiado la estructura factorial del MFE han utilizado muestras de sujetos con patología y el único estudio que se ha realizado con población sin patología se llevó a cabo con estudiantes. Este hecho nos ha llevado a plantear esta investigación con una muestra más generalizable: sujetos trabajadores adultos y jóvenes en activo y sin deterioro cognitivo. Actualmente hay numerosos trabajos que investigan las relaciones de los cuestionarios de olvidos cotidianos y las preguntas de quejas de memoria con variables como el rendimiento objetivo de la memoria, la atención y otros predictores, fundamentalmente variables de tipo afectivo (la depresión y la ansiedad sobre todo), variables de personalidad, etc. Sin embargo, respecto al MFE, no hemos encontrado ningún estudio que relacione los factores obtenidos mediante análisis factorial con este tipo de variables predictoras. Nuestra investigación añade por lo tanto, además del estudio de la estructura factorial del MFE, la relación de estos factores con algunas de las variables que actualmente se consideran importantes en este campo.

El objetivo de este trabajo es, por lo tanto, estudiar la estructura factorial del Cuestionario de Fallos de Memoria de la Vida Cotidiana, MFE, y analizar la relación de estos factores con el rendimiento en memoria objetiva, variables de tipo afectivo y con preguntas generales de quejas de memoria.

\section{Método}

\section{Participantes}

La muestra estuvo formada por 647 participantes, con un rango de edad de 19 a 64 años, media 40.08, DT = 10.03; el $26.9 \%$ eran varones. Todos ellos eran trabajadores de una empresa de servicios y pertenecían a todas las categorías de la empresa, desde personal de recepción y administrativos, hasta técnicos superiores. Todos ellos estaban activos e interesados en los temas de memoria. Sólo se admitieron para el estudio aquellos participantes que no presentaban patología neurológica ni psiquiátrica que pudiera comprometer los resultados. Se descartó esta patología mediante observación clínica y una escala de ansiedad y depresión. Las pruebas y la valoración de los sujetos se realizaron por profesionales de psicología y psiquiatría. Se les administraron diversas pruebas en el contexto de una valoración previa a varias sesiones de educación para la salud, proporcionada por la empresa, y a la que acudieron como voluntarios sin ningún tipo de incentivos. Dado que todo el proceso se desarrolló en un ámbito laboral, en todo momento se han guardado las normas de protección de datos de carácter personal. Los protocolos completados sólo se podían identificar mediante una clave creada por el propio participante y sólo conocida por éste. 1.

Las características de la muestra se presentan en la Tabla
Tabla 1. Descriptivos de edad y estudios.

\begin{tabular}{llllll}
\multicolumn{2}{l}{ CARACTERISTICAS: $N=647$} & & \\
\hline EDAD & $n$ & $\%$ & ESTUDIOS & $n$ & $\%$ \\
\hline $19-29$ & 115 & 17.8 & Estudios primarios & 37 & 5.7 \\
$30-39$ & 179 & 27.7 & Bachiller elemental & 109 & 16.9 \\
$40-49$ & 237 & 36.6 & Estudios medios & 207 & 32.0 \\
$50-64$ & 116 & 17.9 & Estudios superiores & 294 & 45.4 \\
\hline
\end{tabular}

\section{Instrumentos}

Se utilizó el Cuestionario de Fallos de Memoria de la Vida Cotidiana (Memory Failures of Everyday-MFE) de Sunderland et al. (1984) (18) para la evaluación subjetiva de la memoria. Consta de 28 ítems sobre situaciones y actividades de la vida diaria. Cada ítem se puntuó en una escala de 0 a 2 puntos ("nunca o rara vez", "algunas veces", "muchas veces"). Se eligió la opción de tres categorías de respuesta porque es la más utilizada en diversos ámbitos para el MFE, tanto en trabajos de investigación como en el ámbito aplicado (Delgado, Fernández y González, 2009; Garamendi, Delgado y Amaya 2010; Landa, 2007; Montejo, Montenegro, Reinoso, de Andrés y Claver, 2003; Quirosa y López, 2009; Requena, López y Ortiz, 2009).

La memoria objetiva, con presentación auditiva y visual, se evaluó mediante:

Listas de palabras de la Escala de Memoria de Wechsler (WMSIII) (Wechsler, 2004). Se le presenta verbalmente al participante una lista de 12 palabras no relacionadas y se le pide que las recuerde en cualquier orden. Esta tarea (presentación y recuerdo posterior) se repite cuatro veces. A continuación, se le presenta una nueva lista de 12 palabras que el participante debe recordar. Por último, se le pide que recuerde la primera lista. Treinta minutos después, se le pide de nuevo que recuerde la primera lista. Se tomó como puntuación de la memoria auditiva, la media de los 7 intentos de recuerdo.

Escenas de Familia de WMS-III. Son cuatro láminas que representan diversas escenas de la vida de una familia; cada una de ellas se presenta durante 10 segundos. Los sujetos tienen que recordar qué personajes están en la lámina y qué lugar ocupan. Se pide un recuerdo inmediato y otro demorado a los 30 minutos. Se tomó como puntuación de la memoria visual, la media del recuerdo inmediato y el demorado.

La evaluación de la ansiedad y la depresión se llevó a cabo utilizando la Escala de Depresión y Ansiedad de Goldberg (EADAC) (Goldberg, Bridges, Duncan-Jones y Grayson, 1988), que fue concebida para permitir la detección de los dos trastornos psicopatológicos más frecuentes. Consta de 18 preguntas que valoran por separado la ansiedad y la depresión. Su punto de corte es $3 / 4$ para ansiedad y $1 / 2$ para depresión. La versión castellana ha demostrado su fiabilidad y validez en el ámbito de la Atención Primaria y tiene una sensibilidad (83.1\%), especificidad (81.8\%) y valor predictivo positivo (95.3\%) adecuados (Montón, Pérez, Campos, García y Lobo, 1993). En el trabajo presente estas pruebas se han empleado en su aspecto cuantitativo. 
Se plantearon también dos preguntas sobre quejas de memoria con respuesta dicotómica ("Sí"/"No"): P1 “¿Tiene Ud. problemas de memoria?", que valora la creencia global del sujeto sobre su memoria, y P2 "Los fallos de memoria ¿alteran algún aspecto de su vida cotidiana?”, que valora su afectación en la vida cotidiana.

\section{Análisis estadístico}

Para el estudio estadístico se han utilizado los programas SPSS versión 15 (SPSS, 2006) y MPlus (Muthén y Muthén, 2006). Se usó el Análisis Factorial Exploratorio (AFE) sobre las correlaciones entre los ítems para descubrir estadísticamente los factores subyacentes. Puesto que las puntuaciones de los ítems no alcanzan el nivel de medida de intervalo, sino que son datos en categorías ordenadas, el AFE se hizo sobre una matriz de correlaciones policóricas (Lancaster y Hamdan, 1964; Olsson, 1979). El método de estimación utilizado fue WLSMV (weighted least squares mean and variance adjusted), un estimador robusto de mínimos cuadrados ponderados, adecuado para datos categóricos (Muthén y Muthén, 2007). No había supuestos teóricos de que los factores resultantes tuviesen que correlacionar. Por estas razones, se permitió una rotación ortogonal de los datos (rotación Varimax). Como criterio de selección de modelo se empleó el ajuste a los datos del modelo más parsimonioso. Como criterios de ajuste se utilizaron Chi cuadrado entre sus grados de libertad (CMIN/DF), RMSEA (Root Mean Square Error of Approximation) y RMSR (Root Mean Square Residual). Para estudiar la consistencia interna se utilizó el alfa de Cronbach (Browne y Cudeck, 1992; Schreiber, Nora, Stage, Barlow y King, 2006; Wheaton, Muthen, Alwin y Summers, 1977).

Para las correlaciones entre los factores y entre estos y las otras variables (incluso las variables dicotómicas) se utilizó la " $r$ " de Pearson. En el caso de las variables ordinales, los resultados fueron muy semejantes a los obtenidos utilizando la "rho" de Spearman. En algunas variables hubo casos "perdidos", que corresponden a sujetos a los que, de modo ocasional, no se les recogió algún dato o no respondieron a alguna prueba. Para todos los estudios se utilizaron los datos directos, nunca datos estandarizados.

\section{Resultados}

La media del MFE fue de $15.25(D T=7.50)$. El rango fue de 0-40. El alfa de Cronbach fue $=.87$ lo que indica una buena consistencia interna. La solución más parsimoniosa con un ajuste adecuado fue la de tres factores, que explicaban el $29.3 \%$ de la varianza. La solución con cuatro factores explicó un $30.99 \%$ de la varianza, es decir, solo un 1.69\% más que la solución de tres factores, por lo que optamos por esta última. Esta solución obtuvo resultados adecuados en los tres índices estudiados. En primer lugar, se empleó un índice de ajuste absoluto, el expresado por el cociente del estadístico Chi-cuadrado entre sus grados de libertad. Se ha propuesto un ratio de menos de 5 para que el ajuste sea razonable (Wheaton et al., 1977). Según este criterio, el ajuste de este modelo en términos globales fue bueno $(C M I N / D F=2.20)$. Se obtuvieron dos índices de residuos, SRMR y RMSEA. El SRMR fue de 0.06, y este indicador suele ser considerado bueno si es inferior a .08 (Schreiber et al., 2006). El RMSEA fue de .04, considerándose normalmente que un buen ajuste viene dado por un valor de RMSE $A$ de menos de .05 (Browne y Cudeck, 1992). La adecuación muestral a este análisis presenta un buen ajuste a los datos $(K M O=.897)$; se rechaza la hipótesis nula de esfericidad (prueba de esfericidad de Bartlett, Chi cuadrado: 3795.379; gl: 378; $p<.001)$.

En la Tabla 2 se presenta la varianza total explicada y en la Tabla 3 se presenta la matriz de componentes rotados (en negrita los valores mayores o iguales que .30).

Tabla 2. Componentes y varianza explicada.

\begin{tabular}{lccc}
\hline Componente & \multicolumn{3}{c}{$\begin{array}{c}\text { Suma de las saturaciones al cuadrado } \\
\text { de la rotación Varimax }\end{array}$} \\
\cline { 2 - 4 } & Total & \% de la varianza & $\%$ acumulado \\
\hline 1 & 4.31 & 15.41 & 15.41 \\
2 & 2.75 & 5.49 & 20.89 \\
3 & 4.20 & 8.40 & 29.29 \\
\hline
\end{tabular}

Método de estimación: WLSMV.

Según estos resultados, se han hallado tres factores.

Factor 1: Recuerdo de Actividades. Hace referencia a la memoria relacionada con actividades tanto con un componente de memoria prospectiva como retrospectiva.

Factor 2: Reconocimiento. Hace referencia al reconocimiento de lugares y personas.

Factor 3: Monitorización de la Comunicación. En este factor se engloban aspectos de monitorización en relación con la comunicación. Únicamente un ítem (el 15) no se articula al factor en el que tiene más peso (Factor 3, con .53) sino a otro factor muy próximo (Factor 1, con .52), dado que se trata de actividades realizadas.

A continuación, se calculó la puntuación de cada sujeto en cada factor, sumando los valores de los ítems que lo componen. Para poder comparar esas puntuaciones, se dividió cada una de ellas entre el número de ítems. De este modo, se puede también obtener una puntuación en cada dimensión para cada sujeto. A partir de estas puntuaciones ponderadas, se calculó la consistencia interna y la fiabilidad de cada factor. Recuerdo de Actividades: alfa de Cronbach $=.795$; fiabilidad dos mitades de Gutman $=.776$. Reconocimiento: alfa de Cronbach $=.595$; fiabilidad dos mitades de Gutman $=.612$. Monitorización de la Comunicación: Alfa de Cronbach $=.808$; frabilidad dos mitades de Gutman = .806. En la Tabla 4 se presentan las correlaciones entre los tres factores y entre éstos y las variables edad, estudios, memoria visual, memoria auditiva, ansiedad y depresión, y preguntas de quejas (P1 y P2). 
Tabla 3. Matriz de componentes rotados.

\begin{tabular}{|c|c|c|c|c|}
\hline Elemento & Media $(D T)$ & Factor 1 & Factor 2 & Factor 3 \\
\hline 1. Olvidar dónde se ponen las cosas & $1.11(.65)$ & .71 & .11 & -.08 \\
\hline 14. Olvidar tareas que hay que hacer & $.48(.59)$ & .63 & -.03 & .42 \\
\hline 7. Olvidar llevarse objetos cotidianos (gafas,...) & $.73(.67)$ & .62 & .16 & .04 \\
\hline 22. Olvidar cosas que hace habitualmente & $.39(.56)$ & .60 & .15 & .36 \\
\hline 24. Olvidar dónde se guardan las cosas & $.39(.58)$ & .58 & .17 & .14 \\
\hline 4. Olvidar un cambio en las actividades & $.75(.62)$ & .56 & .25 & .26 \\
\hline 15. Olvidar actividades importantes del día anterior & $.33(.53)$ & .52 & .09 & .53 \\
\hline 18. Olvidarse de dar un recado & $.67(.60)$ & .51 & .03 & .32 \\
\hline 27. Realizar una acción dos veces por error. & $.21(.44)$ & .48 & .27 & .23 \\
\hline 5. Tener que comprobar si ha hecho algo & $1.6(.62)$ & .45 & .14 & .12 \\
\hline 25. Perderse en un sitio muy conocido & $.20(.47)$ & .11 & .82 & .15 \\
\hline 26. Perderse en un sitio poco conocido & $.84(.60)$ & .17 & .64 & -.03 \\
\hline 2. No reconocer lugares donde se ha estado antes & $.37(.60)$ & .26 & .48 & .27 \\
\hline 19. Olvidar datos personales importantes (dónde se vive,...) & $.04(.19)$ & .16 & .46 & .23 \\
\hline 11. No reconocer a parientes o amigos & $.17(.42)$ & .20 & .39 & .32 \\
\hline 23. No reconocer las caras de famosos & $.32(.58)$ & .17 & .37 & .31 \\
\hline 10. Divagar en una conversación & $.58(.65)$ & .11 & .06 & .62 \\
\hline 17. Olvidar la trama de la historia que está leyendo & $.38(.55)$ & .02 & .34 & .61 \\
\hline 9. Empezar a leer algo sin recordar que ya se había leído & $.32(.54)$ & .04 & .30 & .56 \\
\hline 16. Olvidar lo que acaba de decir & $.56(.57)$ & .31 & .04 & .53 \\
\hline 6. Olvidar cuándo ocurrió algo & $.74(.66)$ & .30 & .21 & .50 \\
\hline 8. Olvidar lo que le dicen & $.87(.63)$ & .47 & .17 & .50 \\
\hline 20. Mezclar-confundir lo que le han dicho & $.67(.59)$ & .38 & .18 & .48 \\
\hline 3. No poder seguir una historia en TV & $.26(.50)$ & .04 & .38 & .46 \\
\hline 28. Repetir preguntas o lo que acaba de contar & $.41(.56)$ & .33 & .21 & .45 \\
\hline 12. Tener dificultad para aprender una nueva habilidad & $.54(.63)$ & .08 & .38 & .43 \\
\hline 13. Tener una palabra "en la punta de la lengua" & $1.25(.59)$ & .32 & .18 & .41 \\
\hline 21. Repetir una anécdota o chiste que ya se ha contado & $.68(.62)$ & .27 & .07 & .39 \\
\hline
\end{tabular}

21. Repetir una anécdota o chiste que ya se ha contado

Tabla 4. Correlaciones de los factores con otras variables del estudio.

\begin{tabular}{|c|c|c|c|}
\hline$\overline{\text { Variables }}$ & $\begin{array}{c}\text { Factor } 1 \\
\text { Recuerdo de Actividades } \\
M(D T)=.61(.35)\end{array}$ & $\begin{array}{c}\text { Factor } 2 \\
\text { Reconocimiento } \\
M(D T)=.32(.29)\end{array}$ & $\begin{array}{c}\text { Factor } 3 \\
\text { Monitorización de de la Comunicación } \\
M(D T)=.60(.32)\end{array}$ \\
\hline Factor 2 Reconocimiento & $.382(* *)$ & & \\
\hline Factor 3 Monitorización & $.572(* *)$ & $.479(* *)$ & \\
\hline Edad & .075 & $.171(* *)$ & $.127(* *)$ \\
\hline Estudios & -.051 & -.077 & $-.144(* *)$ \\
\hline Memoria visual & .034 & $-.118\left(^{*}\right)$ & .019 \\
\hline Memoria auditiva &,- 051 & $-.139\left(^{*}\right)$ & -.065 \\
\hline Ansiedad y depresión & $.261(* *)$ & $.118\left(^{*}\right)$ & $.247(* *)$ \\
\hline P1 Problemas de memoria & $-.302(* *)$ & $-.168(* *)$ & $-.308(* *)$ \\
\hline P2 Afectación Vida cotidiana & $-.387(* *)$ & $-.169(* *)$ & $-.301(* *)$ \\
\hline
\end{tabular}
${ }^{*} p<.01 ;{ }^{* *} p<.001$

Los dos factores que tienen una puntuación media entre elementos más elevada son el Factor Recuerdo de Actividades (0.67) y el factor Monitorización de la Comunicación (0.61). El factor reconocimiento tiene una media menor (0.32). Ello indicaría que los olvidos a los que se refieren los dos primeros son más frecuentes. Además, estos dos factores muestran una correlación más elevada entre sí que con Reconocimiento. Podemos observar también que la edad muestra correlaciones positivas y significativas con Reconocimiento (a más edad, más olvidos ligados a fallos del reconocimiento) y con Monitorización de la Comunicación, pero no con Recuerdo de Actividades. Sin embargo los estudios muestran una correlación negativa significativa con Monitorización de la Comunicación (a más estudios menos olvidos cotidianos relacionados con la Monitorización de la Comunicación), pero no con Recuerdo de actividades ni con Reconocimiento. El factor afectivo (ansiedad y depresión) correlaciona de manera más clara con Recuerdo de Actividades y Monitorización de la Comunicación que con Reconocimiento. Respecto a la correlación con las dos preguntas de quejas, (P1 o la percepción global de tener problemas de memoria y P2 o la percepción de que éstos afectan a la vida cotidiana) los factores que tienen mayor correlación con estas preguntas son los más frecuentes, el de Recuerdo de Ac- 
tividades y el de Monitorización de la Comunicación. Observamos también que la memoria visual y la auditiva correlacionan de modo negativo con el Reconocimiento, es decir que, a menor puntuación en memoria visual y auditiva, más olvidos ligados a reconocimiento.

\section{Discusión}

Hemos realizado el análisis factorial del MFE con una muestra de 647 personas adultas entre 19 y 64 años. Hemos encontrado una buena consistencia interna y tres factores que explican aproximadamente el $30 \%$ de la varianza. Los tres factores, Recuerdo de Actividades, Reconocimiento y Monitorización de la Comunicación, presentan concordancias y diferencias con los obtenidos por otros autores.

El factor 1 (Recuerdo de Actividades) hace referencia a olvidos relacionados con guardar cosas, cambio en actividades, cosas que hay que hacer, etc. Es un factor muy homogéneo. Todos los olvidos tienen que ver con algo que se ha hecho o que hay que hacer. Son olvidos relacionados con la memoria retrospectiva (por ejemplo: "Olvidar dónde ha puesto alguna cosa") o prospectiva (como "Olvidar decir a alguien algo importante"). Tiene elementos de memoria declarativa episódica ("Olvidar detalles de lo que hizo el día anterior"). El componente atencional también es importante ("Olvidar dónde ha puesto alguna cosa", "Comprobar si se ha hecho algo que tenía intención de hacer" o "Realizar una acción dos veces por error"). También son de notar los elementos de planificación e intencionales. Es, por lo tanto, un factor que se relaciona con varios tipos de memoria y a la vez con las funciones ejecutivas (planificación, monitorización e intencionalidad).

Este factor de Recuerdo de Actividades correlaciona de modo importante con el factor Monitorización de la Comunicación. Además, los dos tienen la puntuación media entre ítems más elevada. Por lo tanto, son los que se relacionan con fallos de memoria que suceden (o se percibe que suceden) con más frecuencia. Sin embargo observamos que Recuerdo de Actividades tiene que ver poco con la edad y los estudios y el rendimiento objetivo de memoria. A su vez, tiene una correlación significativa con ansiedad y depresión y con las preguntas de quejas. Es el factor que tiene una correlación más elevada con la pregunta (P2) que trata de la visión subjetiva global de si se altera o no la vida cotidiana por los fallos de memoria. Cabe deducir, pues, que las actividades que recoge este factor son las que presentan una asociación mayor con esta percepción; más incluso que los fallos de reconocimiento. De estas relaciones deducimos que es un factor con gran componente subjetivo y relacionado con percepciones de bajo rendimiento, que van ligadas a factores afectivos, pero que tienen menos que ver con el rendimiento real de la memoria.

Para otros autores, este factor de Recuerdo de Actividades se distribuye en diversos componentes: comunicación expresiva, cambios en la rutina, memoria prospectiva o memoria general, problemas en la atención o despistes, recupe- ración, monitorización de tareas y conversaciones, etc. El más semejante a nuestro factor es el de Cornish (2000) que tiene un factor que llama "Actividades" que comprende varios de nuestros ítems, aunque no todos. Todos los ítems, cuatro, que corresponden al factor de Efklides et al. (2002) "cambios en la rutina diaria" están en nuestro factor Recuerdo de Actividades. Parte de nuestros ítems son para Richardson (1996) elementos de su factor "atención/ monitorización". Pueden observarse, pues, las diferencias, pero también los importantes solapamientos con los factores de otros autores.

El factor 2 o Reconocimiento reúne todos los ítems que tienen que ver con reconocer en distintas situaciones caras y lugares o rutas. Este factor implica procesos de tipo visual, de recuperación de la información a través de claves y contextos, aspectos de familiaridad, distractores, etc., todos ellos relacionados con las tareas de reconocimiento. La media de este factor, comparándola con los otros dos, indica que se trata de olvidos mucho menos frecuentes en esta muestra de adultos sin patología. Sabemos que cuando hay deterioro cognitivo se suele afectar antes el recuerdo que el reconocimiento. Por lo tanto, es lógico que, en estos sujetos que no padecen deterioro, sea éste el factor que presente las medias más bajas. Sin embargo, observamos que tiene una correlación mayor que los otros factores con la edad, que con frecuencia se asocia a un deterioro de la memoria, y con el rendimiento total de memoria; a la vez que menor correlación con los aspectos afectivos y con la percepción subjetiva de problemas de memoria. Es, por lo tanto, el factor que indica mayor alteración real de la memoria. La desorientación espacial y el fallo del reconocimiento son síntomas de deterioro cognitivo y de enfermedad de Alzheimer (Cahn et al., 1998; Hirono et al., 1998). El hecho de que un cuestionario de olvidos cotidianos como el MFE incluya este factor indica que es un instrumento que podría utilizarse para evaluar las quejas en el deterioro cognitivo.

Este factor está recogido con particularidades en otros autores. Si lo comparamos, podemos observar que en Richardson (1996) este componente se divide en dos, el de reconocimiento de caras y el de rutas, aunque el autor también tiene otros ítems en el factor "rutas" que no se relacionan de modo expreso con rutas, como "tener dificultades para seguir una historia por televisión” y "olvidar un cambio en sus actividades diarias". En Cornish (2000), los ítems de reconocimiento se encuentran en dos factores "monitorización de tareas" y "memoria espacial". En Efklides et al. (2002), se encuentran en "memoria visoespacial" y en "memoria episódica y de caras"; los ítems de "memoria visoespacial" de este último autor corresponden todos a nuestro factor reconocimiento. En los dos últimos autores citados, estos componentes tienen otras preguntas no relacionadas directamente con los aspectos de reconocimiento de caras o lugares. Dejando aparte las importantes similitudes, no encontramos una explicación clara para estas diferencias entre autores, aunque parece que nuestro factor es el más uniforme. 
El factor 3 comprende ítems con contenidos cuyo denominador común se refiere a la monitorización y control de la comunicación, tanto expresiva como receptiva. En ellos está implicada la memoria de trabajo en su aspecto de planificación y monitorización de tareas ("dificultades en seguir una historia por televisión", "no seguir una trama al leer", "mezclar cosas que le han dicho"). Podríamos decir que es el factor más "cultural" pues los olvidos tienen que ver con lectura, lenguaje, comprensión de la información recibida, etc. Para otros autores estos ítems están encuadrados en componentes como "monitorización de tareas" y "monitorización de conversaciones" (Cornish, 2000), "comunicación receptiva y expresiva" (Richardson, 1996), "memoria prospectiva", "memoria general", "aprendizaje nuevo" y "repetición de respuestas" (Efklides et al., 2002) y "atención" (Royle y Lincoln, 2008). En estos olvidos se involucran áreas como la memoria a corto plazo y la memoria de trabajo, así como componentes atencionales, de lenguaje y otros. Hay también, como en el factor 1 , elementos de funciones ejecutivas.

El factor 3 es un factor próximo al de Recuerdo de Actividades. Se correlaciona de modo significativo con la variable ansiedad y depresión, con los aspectos subjetivos de percepción de problemas de memoria y su influencia en la vida cotidiana, pero también se relaciona con la edad aunque no con el rendimiento de memoria objetiva. Puede considerarse como un factor en gran parte subjetivo. El hecho de relacionarse de modo significativo con los estudios nos indica la influencia del aspecto educacional o cultural en los olvidos contenidos en este factor.

Si tuviéramos que hacer un juicio global que explicara y justificara las diferencias de este estudio con los resultados obtenidos por los demás autores que han realizado análisis de factores, probablemente tendríamos que centrarnos en el origen de las muestras y los objetivos secundarios de los distintos trabajos. Efklides et al. (2002) estudian sujetos sanos y otros con Alzheimer, siendo uno de sus objetivos analizar las relaciones con otras pruebas de memoria; Royle y Lincoln (2008) escogen sujetos normales y otros con patología (ictus y esclerosis múltiple), siendo un objetivo fundamental de estos autores construir una versión más breve del MFE. Richardson (1996) toma pacientes de esclerosis múltiple y sus familiares; Cornish (2000) hace su estudio con estudiantes. Estas muestras condicionan los resultados y marcan diferencias entre ellos y con nuestro trabajo.

\section{Conclusiones}

Las respuestas a este cuestionario representan cómo los adultos viven la experiencia diaria del funcionamiento de su memoria. Las correlaciones de los factores con los resultados de las otras pruebas administradas a los participantes en el estudio nos indican aspectos de tipo objetivo (las relaciones de los factores con las pruebas objetivas) y de tipo subjetivo (las relaciones de los factores con las pruebas subjetivas), aunque estaríamos de acuerdo con Richardson (1996) en que hay un elemento que se encuentra en todas las respuestas al Cuestionario de Fallos de Memoria de la Vida Cotidiana: el trastorno subjetivo de la memoria.

Los factores se pueden interpretar y explicar de acuerdo con los procesos teóricos de memoria subyacentes y según los subsistemas teóricos clásicos, pero estas interpretaciones quizá no sean del todo satisfactorias, ni expliquen totalmente los factores. En los factores hallados, encontramos dominios muy estudiados desde un punto de vista teórico, como la memoria de trabajo, la memoria episódica, el fenómeno de "reconocimiento" de caras y lugares; hay otros aspectos relacionados con la memoria cotidiana tanto retrospectiva como prospectiva. En algunos de estos factores se implican a la vez procesos de memoria, tanto verbal como visual, y de atención. En casi todos hay también aspectos relacionados con las funciones ejecutivas, y en muchos se implica el área del lenguaje.

Otro aspecto que nos muestra el análisis de los factores del MFE es que estos olvidos nos pueden indicar alteraciones de diversa gravedad. Las alteraciones más graves serían las relacionadas con alteraciones del reconocimiento (Factor 2: no reconocer las caras de amigos o parientes, perderse en un sitio donde ha estado antes con frecuencia,...). Seguirían en gravedad las alteraciones en los que se implica la memoria episódica de hechos recientes (Factor 3: hacer dos veces la misma pregunta, no poder seguir una historia, olvidar lo que se acaba de decir,...). Los menos graves serían los que se refieren a olvidos de actividades (Factor 1: olvidar llevarse objetos, olvidar dónde se guardan las cosas,...). Habría que añadir que un número mayor de olvidos dentro de cada uno de los factores, y una mayor frecuencia dentro de cada uno de esos olvidos, también indicarían mayor afectación a la vida diaria y, por lo tanto, mayor gravedad.

Esta progresión en la gravedad de los olvidos presentes en cada uno de los tres factores, del modo que hemos indicado, está apoyada por numerosos estudios. El aspecto mejor fundamentado es la mayor gravedad de las alteraciones del reconocimiento respecto a las de recuerdo (Light, 1991; Smith, Del Sala, Logie y Maylor, 2000). Una de las explicaciones que se da a este hecho es que, en las tareas de reconocimiento, se aportan las claves de recuperación que están presentes en el contexto, haciendo más fáciles esas tareas que las de recuerdo libre (Bennett, Golob, Parker y Starr, 2006). En la Escala de Deterioro Global (GDS) de Reisberg (Reisberg, Ferris, de León y Crook, 1982), que divide el deterioro cognitivo en siete fases (de menor a mayor deterioro) y que es la más utilizada en este campo, el factor 1 (recuerdo de actividades tanto en el sentido retrospectivo como prospectivo) está recogido en la fase 2 , mientras que los recuerdos relacionados con la monitorización se encuentran sobre todo en la fase 3 (Deterioro Cognitivo Leve). Aumentaría la gravedad de estos últimos el hecho de que son olvidos muy recientes (olvidar lo que se acaba de decir, mezclarconfundir lo que le han dicho, etc.) y que tienen un componente de disfunción ejecutiva (Amariglio et al., 2011). Las alteraciones en el reconocimiento se encuentran tanto en la fa- 
se 3 de GDS (perderse en un lugar no familiar) como en la fase 4 (no reconocer las caras de familiares o amigos), indicando esta última fase Deterioro Cognitivo Moderado.

Una de las limitaciones de nuestro estudio es la posibilidad de generalizar los resultados. Los sujetos de estudio no se han extraído mediante un muestreo aleatorizado y estratificado de toda la población con características semejantes. Para que estos resultados sean plenamente generalizables, deberán realizarse otros estudios con un tamaño de muestra adecuado según la población objeto, aleatorizada, con sujetos elegidos según estratos de edad, sexo y estudios, etc. Otra limitación de nuestro trabajo es que se trata de una muestra de adultos jóvenes, no de mayores. Esto limita la generalización de los resultados aquí obtenidos a esta población de mayores, para la que es muy útil la utilización del MFE, tanto en lo que respecta a la valoración de quejas de memoria como en lo que respecta a la evaluación de los resultados de las intervenciones de los programas de entrenamiento de memoria, que se están desarrollando cada vez más. Por otra parte, las dos preguntas generales de quejas no

\section{Referencias}

Abdulrab, K. y Heun, R. (2008). Subjective Memory Impairment. A review of its definitions indicates the need for a comprehensive set of standardised and validated criteria. European Psychiatry, 23, 321-330. http://dx.doi.org/10.1016/j.eurpsy.2008.02.004

Amariglio, R. E., Mary K. Townsend, M. K., Grodstein, F., Sperling, R. A. y Rentz, D. M. (2011) Specific Subjective Memory Complaints in Older Persons May Indicate Poor Cognitive Function. The Journal of American Geriatrics Society, 59, 1612-1617.

Benedet, M. J. y Seisdedos, N. (1996). Evaluación clínica de las quejas de memoria en la vida cotidiana. Buenos Aires: Editorial médica Panamericana.

Bennett, I. J., Golob, E. J., Parker, E. S. y Starr, A. (2006). Memory Evaluation in Mild Cognitive Impairment using Recall and Recognition Tests. Journal of Clinical and Experimental Neuropsychology, 28, 1408-1422. http://dx.doi.org/10.1111/j.2044-8260.1980.tb00946.x

Broadbent, D. E., Cooper, P., Fitzgerald, P. y Parkes, K. R. (1982). The Cognitive Failures Questionnaire (CFQ) and its correlates. British Journal of Clinical Psychology, 21,1-16. http://dx.doi.org/10.1111/j.20448260.1982.tb01421.x

Browne, M. W. y Cudeck, R. (1992). Alternative ways of assessing model fit. Sociological Methods y and Research, 21, 230.

Cahn, D. A., Sullivan, E. V., Shear, P. K., Marsh, L., Fama, R., Lim, K. O. et al. (1998). Structural MRI correlates of recognition memory in Alzheimer's disease. Journal of the International Neuropsychological Society, 4, 106-114.

Cavallini, E., Pagnin, A. y Vecchi, T. (2003). Aging and everyday memory: the beneficial effect of memory training. Archives of Gerontology and Geriatrics, 37, 241-257. http://dx.doi.org/ 10.1016/S01674943(03)00063-3

Cornish, I. M. (2000). Factor structure of the everyday memory questionnaire. British Journal of Psychology, 91, 427-438. http://dx.doi.org/10.1348/000712600161916

Crook, T., Bartus, R. T., Ferris, S. H., Whitehouse, P., Cohen, G. D. y Gershon, S. (1986). Age-associated memory impairment: Proposed diagnostic criteria and measures of clinical change-report of a National Institute of Mental Health Work Group. Developmental Neuropsychology, 2, 261-276. http://dx.doi.org/10.1080/ 87565648609540348

Crook, T. H., Feher, E. P. y Larrabee, G. J. (1992). Assessment of memory complaint in age-associated memory impairment: the MAC-Q. International psychogeriatrics, 4, 165-176. están validadas. No conocemos ningún trabajo que haya validado preguntas de quejas, pero hemos aplicado las más utilizadas en las investigaciones sobre este tema.

Este trabajo tiene implicaciones prácticas, tanto para la investigación como para la clínica. Los olvidos de la vida cotidiana pueden valer para enriquecer la investigación sobre los modelos y el funcionamiento de la memoria. Las respuestas y los factores que subyacen deben ser tenidos en cuenta en el desarrollo de esta investigación, pues aportarán realidad y, por lo tanto, fiabilidad y validez. En la consulta diaria, con pacientes que refieren quejas de memoria y olvidos cotidianos, una de las cuestiones que constantemente nos planteamos es hasta qué punto es grave un olvido que manifiesta un individuo o su familia. Pues bien, el MFE y el análisis de los factores que lo integran nos pueden valer como elemento de juicio para interpretar mejor la gravedad de las alteraciones de un paciente, teniendo también en cuenta que, al manifestarse como quejas de memoria, intervienen otros elementos distintos de la alteración objetiva de la memoria (Montejo, Montenegro, Fernández, y Maestú, 2011).

Delgado, M. L., Fernández, S. y González-Marqués, J. (2009). Evaluación de los problemas de memoria cotidiana en personas mayores. Adaptación española del Test de Memoria Conductual Rivermead-III. Memoria final, proyecto 119/07. Madrid: Universidad Complutense de Madrid. Facultad de Psicología.

Dixon, R. A. y Hultsch, D. F. (1983). Metamemory and memory for text relationships in adulthood: A cross-validation study. Journal of Gerontology, 38, 689.

Drysdale, K., Shores, A. y Levick, W. (2004). Use of the Everyday Memory Questionnaire with children. Child Neuropsychology, 10, 67-75. http://dx.doi.org/10.1080/09297040490911087

Efklides, A., Yiultsi, E., Kangellidou, T., Kounti, F., Dina, F. y Tsolaki, M. (2002). Wechsler Memory Scale, Rivermead Behavioral Memory Test, and Everyday Memory Questionnaire in healthy adults and Alzheimer patients. European Journal of Psychological Assessment, 18, 63-77. http://dx.doi.org/10.1027//1015-5759.18.1.63

Fernández Ballesteros, R., Izal, M. y Montorio, I. (1991). Evaluación e intervención psicológica en la vejez: Barcelona: Martínez Roca.

Garamendi, F., Delgado, D. A. y Amaya, M. A. (2010) Programa de entrenamiento cognitivo en adultos mayores. Revista Mexicana de Medicina Física y Rehabilitación, 22, 26-31.

García-Martínez, J. y Sánchez Cánovas, J. (1994). Adaptación del cuestionario de fallos cognitivos de Broabdent, Cooper, Fitzgerald y Parkes (CFQ cognitive failure questionnarie). Análisis y modificación de conducta, 20, 727-752.

García-Martínez, J. y Sánchez-Cánovas J. (1993) Adaptación del cuestionario de fallos de memoria en la vida cotidiana (MFE). Boletín de Psicología, 43, 89-105.

Goldberg, D., Bridges, K., Duncan-Jones, P. y Grayson, D. (1988). Detecting anxiety and depression in general medical settings. British Medical Journal, 297, 897-899. http://dx.doi.org/10.1136/bmj. 297.6653.897

Goldstein, L. y Polkey, C. (1992). Everyday memory after unilateral temporal lobectomy or amygdalo-hippocampectomy. Cortex, 28, 189-201.

Hermann, D. J. (1982). Know thy memory: The use of questionnaires to assess and study memory. Psychological Bulletin, 92, 434-452.

Hirono, N., Mori, E., Ishii, K., Ikejiri, Y., Imamura, T., Shimomura, T. et al. (1998). Hypofunction in the posterior cingulate gyrus correlates with disorientation for time and place in Alzheimer's disease. Journal of Neurology, Neurosurgery and Psychiatry, 64, 552-554.

Jorm, A.F., Christensen H., Korten A.E., Jacob PA. y Henderson, S. A. (2001). Memory Complaints as a precursor of memory impairment in 
older people: a longitudinal analysis over 7-8 years. Psychological Medicine, 31, 441-449.

Lancaster, H. y Hamdan, M. (1964). Estimation of the correlation coefficient in contingency tables with possibly nonmetrical characters. Psychometrika, 29, 383-391.

Landa, P. J. (2007). Taller de Entrenamiento de Memoria, Informe. Servicios psicológicos. Vitoria.

Larrabee, G. y Crook, T. (1988). A computerized everyday memory battery for assessing treatment effects. Psychopharmacology bulletin, 24, 695-697.

Light, L. L. (1991). Memory and aging: Four hypotheses in search of data. Annual Review of Psychology, 42, 333-376.

Montejo, P., Montenegro, M., Reinoso, A., De Andrés, M. y Claver, M. D. (1999). Estudio de la eficacia de un programa de entrenamiento de memoria multicéntrico para mayores de 60 años. Revista española de Geriatría y Gerontología, 34, 199-208.

Montejo, P., Montenegro, M., Reinoso, A. I., de Andrés, M. E. y Claver, M. D. (2003). Manual práctico de Evaluación y Entrenamiento de Memoria. Método $U M A M$. Madrid: Díaz de Santos.

Montejo, P., Montenegro, M., Fernández, M. A. y Maestú, F. (2011). Subjective memory complaints in the elderly: Prevalence and influence of temporal orientation, depression and quality of life in a populationbased study in the city of Madrid. Aging and Mental Health, 15, 85-96. http://dx.doi.org/10.1080/13607863.2010.501062

Montón, C., Pérez , M., Campos, R., García, J. y Lobo, A. (1993). Escalas de ansiedad y depresión de Goldberg: una guía de entrevista eficaz para la detección del malestar psíquico. Atención primaria, 12, 345-349.

Muthén, L. y Muthén, B. (2006). Mplus: Statistical analysis with latent variables (Version 4.1)[Computer software]. Los Angeles, CA: Authors.

Muthén, L. y Muthén, B. (2007). MPlus User's guide. Fifth edition. Muthén y Muthén.

Olsson, U. (1979). Maximum likelihood estimation of the polychoric correlation coefficient. Psychometrika, 44, 443-460.

Petersen, R. (2004). Mild cognitive impairment as a diagnostic entity. Journal of Internal Medicine, 256, 183-194.

Ponds R. W., Commissaris K. J. y Jolles, J. (1997). Prevalence and covariates of subjective forgetfulness in a normal population in the Netherlands. International Journal of Aging and Human Development, 45, 207-221

Quemada, J. I., Muñoz Céspedes, J. M., Ezkerra, J., Ballesteros, J., Ibarra, N. y Urruticoechea, I. (2003). Outcome of memory rehabilitation in traumatic brain injury assessed by neuropsychological tests and questionnaires. The Journal of head trauma rehabilitation, 18, 532-540.

Quirosa, T. y López, F.J. (2009). Aplicación de la terapia de aceptación y compromiso a las quejas subjetivas de memoria en personas mavores de 60 años. Comunicación presentada al IX Congreso Nacional de Organizaciones de Mayores, Sevilla.

Reid, L. M. y MacLullich, A. M. J. (2006) Subjective memory complaints and cognitive impairment in older people. Dementia Geriatrics and Cognitive Disorders, 22, 471-485
Reisberg, B., Ferris, S.H., de León, M.J. y Crook, T. (1982). The global deterioration scale for assessment of primary degenerative demenctia. American Journal of Psychiatry, 139, 1136-1139.

Requena, C., López, V. y Ortiz, T. (2009). Satisfacción con la vida en relación con la funcionalidad de las personas mayores activas. Actas Españolas de Psiquiatría, 37, 61-67.

Richardson, J. (1996). Memory impairment in multiple sclerosis: reports of patients and relatives. The British Journal of Clinical Psychology/the British Psychological Society, 35, 205-219. http://dx.doi.org/10.1111/j.2044-8260. 1996.tb01177.x

Royle, J. y Lincoln, N. B. (2008). The Everyday Memory Questionnairerevised: Development of a 13-item scale. Disability and Rehabilitation, 30 , 114-121. http://dx.doi.org/10.1080/09638280701223876

Schreiber, J. B., Nora, A., Stage, F. K., Barlow, E. A. y King, J. (2006). Reporting structural equation modeling and confirmatory factor analysis results: A review. The Journal of Educational Research, 99, 323-338.

Seltzer, B., Vasterling, J. J., Hale, M. A. y Khurana, R. (1995). Unawareness of memory deficit in Alzheimer's disease: relation to mood and other disease variables. Cognitive and Behavioral Neurology, 8, 176.

Smith, G., Del Sala, S., Logie, R.H. y Maylor, E.A (2000). Prospective and retrospective memory in normal ageing and dementia: A questionnaire study, Memory, 8, 311-321.

SPSS Inc.Chicago Il 60606-6412 EE.UU.

Sunderland, A., Harris, J. E. y Baddeley, A. D. (1983). Do laboratory test predict everyday memory? A neuropsychological study. Journal of Verbal Learning and Verbal Behavior, 22, 341-357. http://dx.doi.org/10.1016/ S0022-5371(83)90229-3

Sunderland, A., Harris, J. E. y Gleave, J. (1984). Memory failures in everyday life following severe head injury. Journal of Clinical and Experimental Neuropsychology, $\quad 6, \quad$ 127-142. http://dx.doi.org/ 10.1080/01688638408401204

Sunderland, A., Watts, K., Baddeley, A. D. y Harris, J. E. (1986). Subjective memory assessment and test performance in elderly adults. The Journal of Gerontology, 41, 376.

Wechsler, D. (2004). Escala de memoria de Wechsler-III: WMS-III: manual técnico. Madrid: TEA Ediciones.

West, R. L. y Sinnott, J. D. (1992). Everyday memory and aging: Current research and methodology: Springer-Verlag.

Wheaton, B., Muthen, B., Alwin, D. F. y Summers, G. F. (1977). Assessing reliability and stability in panel models. Sociological methodology, 8, 84-136.

Wilson, B., Cockburn, J. y Baddeley, A. (1985). The Rivermead Behavioural Memory Test, Bury St. Edmunds: Thames Valley Test Company.

Zelinski, E. Gilewski, M. y Thompson, L. (1980). Do laboratory test relate to self-assessment of memory ability in the young and old? En L.W. Poon, J.L. Fozard, L.S. Cermak, D., Arenberg, \& L.W. Thompson (Eds.), New directions in memory and aging: Proceedings of the George A. Talland Memorial Conference (pp. 519-544). Hillsdale, N.J.: Erlbaum.

(Artículo recibido: 22-06-2011 revisado: 20-02-2013, aceptado: 04-04-2013) 